\title{
Echinorhinus cookei Pietschmann, 1928 (Chondrichthyes: Squaliformes, Echinorhinidae), primer registro en aguas de El Salvador, Pacífico oriental tropical
}

\author{
Echinorhinus cookei Pietschamnn, 1928 (Chondrichthyes: Squaliformes, Echinorhinidae), \\ first record in El Salvador waters, Tropical Eastern Pacific \\ José R. Rojas ${ }^{1}$, Claudia I. Fuentes ${ }^{2}$ y Numa R. Hernández ${ }^{2}$ \\ ${ }^{1}$ Apartado Postal 20-4200, Naranjo, Alajuela, Costa Rica \\ ${ }^{2}$ Unidad de Investigación y Desarrollo, Pesquera del Sur S.A. de C.V, Acajutla, Sonsonate, El Salvador \\ rrojasm@ice.go.cr
}

\begin{abstract}
The first record of juveniles of prickly shark Echinorhinus cookei Pietschmann, 1928 (Chondrichthyes: Echinorhinidae), in El Salvador is communicated on the basis of two specimens: a male, $52 \mathrm{~cm}$ TL and a female $34.6 \mathrm{~cm}$ TL, captured in waters between Puerto de Acajutla and Puerto
\end{abstract}

La Libertad, El Salvador, Central America. The specimens are deposited in the Museo de Historia Natural de El Salvador, under ichthyological catalogue number MHNES 40-726.

Key words: Pisces, prickly shark, port, Pacific Ocean
Se reporta por primera vez la captura de dos ejemplares de Echinorhinus cookei Pietschmann, 1928 atrapados en aguas del Pacífico oriental salvadoreño, entre el puerto de Acajutla y puerto La Libertad, El Salvador. El primer hallazgo, un ejemplar macho de $52 \mathrm{~cm}$ de longitud total, ocurrió el 12 de marzo de 2005 a los 1256’80”N y $89^{\circ} 40^{\prime} 82^{\prime}$ 'W. La captura se hizo entre las $12: 50$ y 13:25 $\mathrm{h}$, a una profundidad de $180 \mathrm{~m}$, en un fondo arenoso; como fauna acompañante se encontraron tres especies de crustáceos: Pleuroncodes planipes (Stimpson, 1860), Mursia gaudichaudii (Milne Edwards, 1837) y Squilla biformes (Bigelow, 1891). El segundo especimen, una hembra de 34,6 cm longitud total, fue capturada el 29 de agosto de 2005, a $12^{\circ} 51^{\prime} 83^{\prime \prime} \mathrm{N}$ y 89³6'18”W, en arrastres diurnos (9:20-9:35 h) aproximadamente a 357 $\mathrm{m}$ de profundidad en fondo arenoso y como parte de la captura de Physiculus nematopus Gilbert, 1891 (Moridae).

Las capturas se hicieron con dos redes de arrastre de 20,25 m de largo, luz de malla de 7,6 cm en alas y cuerpo y de 5,1 cm en el copo. Los arrastres se realizaron entre las 05:00 y las 19:00 h, con un tiempo efectivo de $15 \mathrm{~min}$, a una velocidad promedio de 2,5 millas náuticas por hora. En el laboratorio, los organismos de cada muestra se separaron por grupos (peces, crustáceos y otros organismos). Los ejemplares de $E$. cookei fueron congelados y fijados en formalina al
$10 \%$ y posteriormente preservados en alcohol al $70 \%$. Los especimenes fueron depositados en el Museo de Historia Natural de El Salvador (MHNES), bajo el catálogo $\mathrm{N}^{\mathrm{o}}$ 40-726. La identificación taxonómica familiar, genérica y específica sigue los lineamientos sugeridos por Garrick (1960), Compagno (1984), Last \& Stevens (1994), Compagno et al. (1995), Compagno \& Niem (1998) y Robertson \& Allen (2002).

En concordancia con estos autores, se determinó que los ejemplares capturados son E. cookei ya que estos tiburones se caracterizan por tener un cuerpo robusto y cilíndrico y cabeza deprimida (Fig. 1). Además tienen cinco ranuras branquiales, espiráculo diminuto por detrás del ojo, sin aleta anal y aleta caudal sin hendidura cerca de la punta, dos pequeñas aletas dorsales de igual tamaño estrechamente cercanas a la aleta caudal, aletas pélvicas grandes, aleta caudal muy asimétrica y con el lóbulo inferior pobremente desarrollados y cuerpo densamente cubierto con dentículos angulares libres con sus bases estrelladas, menores de $5 \mathrm{~mm}$ de diámetro, atributos que lo diferencian de Echinorhinus brucus (Bonnaterre, 1788). Los dientes de los ejemplares capturados son similares en ambas mandíbulas, comprimidos y en forma de placas, cada diente tiene tres puntas grandes y hasta tres pequeñas. Más detalles sobre las características morfométricas se presentan en la Tabla 1. 


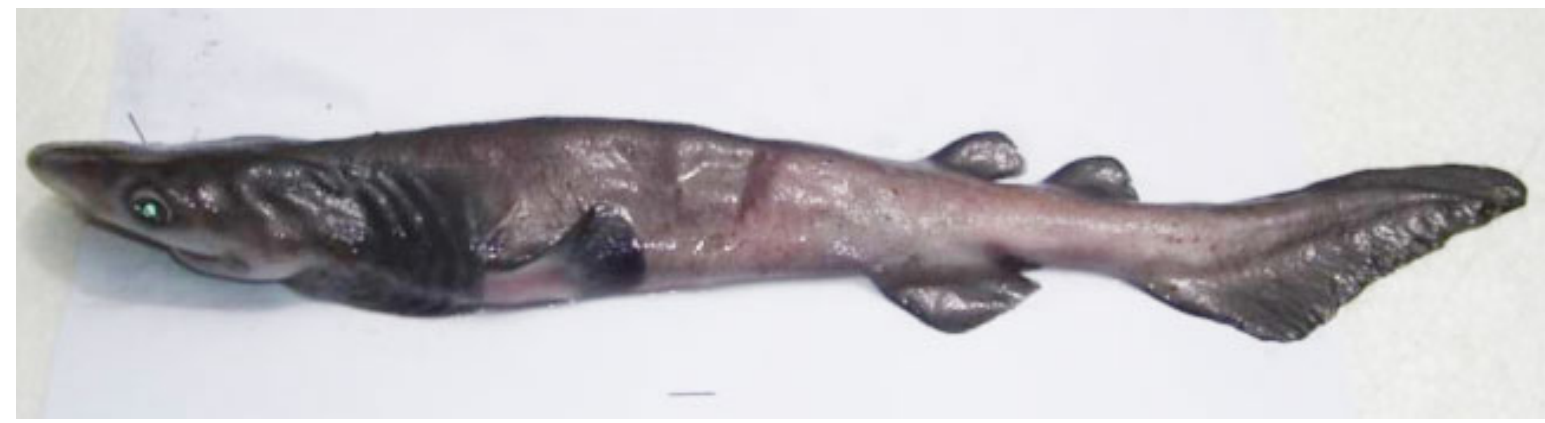

Figure 1

Echinorhinus cookei, 520 mm longitud total, El Salvador, Centro América

Echinorhinus cookei, $520 \mathrm{~mm}$ of total length, El Salvador, Central America

Tabla 1

Medidas de los ejemplares de Echinorhinus cookei (MHNES 40-726)

Measurements of samples of Echinorhinus cookei (MHNES 40-726)

\begin{tabular}{lrr}
\hline \multicolumn{1}{c}{ Dimensión } & $\begin{array}{c}\text { Hembra } \\
(\mathrm{mm})\end{array}$ & $\begin{array}{c}\text { Macho } \\
(\mathrm{mm})\end{array}$ \\
\hline Longitud total & 345 & 520 \\
Longitud preorbital & 25 & 37 \\
Longitud hendiduras branquiales & 81 & 122 \\
Longitud cabeza & 100 & 150 \\
Longitud hasta primera dorsal & 191 & 288 \\
Longitud hasta segunda dorsal & 221 & 333 \\
Longitud precaudal & 244 & 368 \\
Longitud interdorsal & 10 & 15 \\
Longitud segunda dorsal y caudal & 10 & 15 \\
Longitud hasta aleta pectoral & 98 & 148 \\
Longitud hasta pélvica & 174 & 262 \\
Longitud pectoral y pélvica & 64 & 94 \\
Longitud aleta pélvica y caudal & 27 & 41 \\
Longitud interorbital & 35 & 53 \\
Ancho de cabeza & 35 & 53 \\
Ancho de tronco & 37 & 56 \\
Ancho pedúnculo caudal & 6 & 9 \\
Longitud entre narinas & 18 & 21 \\
Longitud de boca & & \\
\hline
\end{tabular}

E. cookei, conocido como tiburón negro espinoso, alcanza hasta $400 \mathrm{~cm}$ y es de hábitat demersal. Es ovovivíparo con más de 114 huevos por litera. El tamaño al nacer es entre $40-45 \mathrm{~cm}$ (nuestros ejemplares son considerados juveniles). Maduran cuando alcanzan $198 \mathrm{~cm}$ (machos) y entre 250 y $300 \mathrm{~cm}$ (hembras) (Last \& Stevens 1994). Se alimentan de una variedad de peces, otros tiburones, pulpos y calamares (Cox \& Francis 1997). Se distribuye ampliamente en el Pacífico; algunos lugares donde han sido reportados son Japón (Taniuchi \& Yanagisawa 1983), Australia (Last \& Steven 1994), Hawai (Chave \& Mundy 1994), Chile (Pequeño 1989 y Brito 2004) y Ecuador (Bearez 1996). Compagno (1984) y Robertson \& Allen (2002) reportan esta especie para el Pacífico subtropical y en el Pacífico Oriental del sur de California al Golfo de California y de Costa Rica a Perú y Malpelo, con un vacío evidente de registros para la nueva zona que se reporta. El hallazgo de estos ejemplares constituye, no solo el primer hallazgo para las aguas salvadoreñas, sino para este sector del Pacífico Oriental; es por eso que la evidencia que se aporta sustituye la hipótesis de la ocurrencia de E. cookei en la parte media del Pacífico centroamericano, para convertirse en un hecho real.

\section{Agradecimientos}

Se agradece a William Bussing por la confirmación taxonómica, a Alberto Olivares Menay por la metodología y a la Pesquera del Sur S.A, Acajutla, Sonsonate, El Salvador, por la oportunidad de estudiar los ejemplares reportados. Se agradece a los revisores anónimos por el excelente trabajo editorial. 


\section{Literatura citada}

Bearez P. 1996. Lista de los peces marinos del Ecuador continental. Revista de Biología Tropical 44: 731-741.

Brito J. 2004. Presencia del tiburón martillo Sphyrna zygaena (Carchariniformes: Sphyrnidae) y nuevo registro del tiburón espinudo Echinorhinus cookei (Squaliformes: Squalidae) en San Antonio, Chile central. Investigaciones Marinas 32(2): 141-144.

Chave E \& B Mundy. 1994. Deep-sea benthic fish of the Hawaiian Archipelago, Cross Seamount and Johnson Atollon. Pacific Science 48: 367-409.

Compagno LJV. 1984. FAO species catalogue. Vol. 4. Sharks of the world. An annotated and illustrated catalogue of sharks species known to date. Part 1 . Hexanchiformes to Lamniformes. FAO Fisheries Synopsis (125) Vol. 4 Pt.1, 249 pp.

Compagno LJV, F Krupp \& W Schneider. 1995. Tiburones. En: Fischer W, Krupp F, Schneider W, Sommer C, Carpenter K \& Niem V (eds), Guía para la identificación de especies para los fines de pesca. Pacífico Centro-Oriental, pp. 647-744. FAO. Rome.

Compagno LJV \& VH Niem. 1998. Echinorhinidae. Bramble sharks. En: Carpenter KE \& Niem VH (eds), FAO identification guide for fishery purposes. The living marine resources of the Western Central Pacific, pp. 12111212. FAO. Rome.
Cox G \& M Francis. 1997. Sharks and rays of New Zealand, 68 pp. Canterbury Univ. Press, England.

Garrick J. 1960. Studies on New Zealand Elasmobrachii. Part $\mathrm{X}$. The genus Echinorhinus with an account of a second species E. cookie. Transactions of the Royal Society of New Zealand 88: 105-117.

Last P \& J Steven. 1994. Sharks and rays of Australia. Fisheries Research \& Development Corporation 1-513 col. pls. 1084.

Pequeño G. 1989. Peces de Chile. Lista sistemática revisada y comentada. Revista de Biología Marina, Valparaíso 24 (2): 1-132.

Robertson R \& G Allen. 2002. Peces costeros del Pacífico oriental tropical: un sistema de información. Instituto Smithsonian de Investigaciones Tropicales, Panamá.

Taniuchi T \& F Yanagisawa. 1983. Occurrence of the prickly shark Echinorhinus cookie at Kumanonada, Japan. Japanese Journal of Ichthyology 29: 465-468.

Recibido el 19 de diciembre de 2005 y aceptado el 17 de abril de 2006 